This divides the charged particle plasma which corotates with the Earth (in fact the upward extension of the ionosphere) from that which is fixed in an EarthSun coordinate system due to the existence of the solar wind. That the waves were not observed within the plasmapause is quite natural, since the ambient density of charged particles is too high for the propogation of electromagnetic waves of low frequencies. The fact, however, that a shadow zone could be traced in the magnetosphere out to distances of 15 Earth radii, showed that the waves must originate in the auroral zones of the atmosphere. Finally, correlation of the observation of particular bursts of radio noise with observations of the auroral regions by the USAF Dapp satellite, which obtains a picture of the auroral oval, showed the electromagnetic radiation to originate in discrete auroral arcs, generally in the evening portion of the auroral zones. Evidently the waves are generated by the intense fluxes of energetic electrons which produce the auroral arcs.

The production process must be efficient, since the energy associated with the electrons is typically $10^{11} \mathrm{~W}$, whereas that in the radio bursts is $10^{9} \mathrm{~W}$. Certainly some cooperative mechanism or plasma instability is required, which is true also in the case of the radio emission from Jupiter. There are other similarities with Jupiter; among others large fluxes of energetic electrons moving along the magnetic field lines are involved in each case, and the frequency of emission also appears to be similar to the electron cyclotron frequency in the emitting region. Gurnett concludes that the types of instability proposed to account for Jovian emissions may also account for the terrestrial radiation.

\section{Catecholamine receptors detected}

from Leslie L. Iversen

THE current vogue for labelling receptor sites has resulted in spectacular progress towards identifying sites for various peptide hormones, neurotransmitters such as acetylcholine and glycine, and drugs, including morphine (see Dismukes, Nature, 252, 442; 1974). Catecholamine receptors, however, have proved more elusive. Various attempts to identify $\beta$-adrenoceptors in tissues such as liver, heart or spleen by measuring the binding of labelled catecholamines or their antagonists to intact cells or to microsomal fractions failed to demonstrate a binding process with the expected properties. Cuatrecasas et al. (Nature, 247, 92) reviewed their own experience in this field at the

\title{
Microwave spectra of molecular ions
}

\author{
from our Chemical Physics Correspondent
}

Microwave or rotational spectra of neutral molecules are straightforward to study, but the difficulties multiply for short-lived molecular ions in the gas phase. This is unfortunate since interstellar conditions, with a comparative absence of collisions, are much more favourable to long life for such species. Indeed the structure $\mathrm{HCO}^{+}$has been tentatively suggested for " $X$-ogen".

Further interest arises since Bunker (Chem. Phys. Lett., 27, 322; 1974) has pointed out that species such as $\mathrm{HD}^{+}$and ${ }^{14} \mathrm{~N}^{15} \mathrm{~N}^{+}$should have quite strong microwave spectra. The corresponding neutral molecules have zero dipole moment, unless extremely small effects are considered, and consequently they have no detectable microwave spectrum. For charged species the dipole moment is no longer independent of the origin of the coordinates to which it is referred. Although if this origin is at the centre of the ion the dipole moment will be equally small, for discussing microwave spectral intensity, as Bunker shows, the appropriate origin is the centre of mass so that species with different isotopes may have quite strong spectra.

beginning of 1974 and persuasively demonstrated that the binding of ${ }^{3} \mathrm{H}$ noradrenaline to such preparations detected a site that recognises primarily the catechol function of the molecule. This site, unlike the $\beta$-adrenoceptor, does not distinguish between the active and inactive stereoisomers of the catecholamines or their antagonists and may be a form of the enzyme catecholO-methyl transferase.

A great deal of advice and criticism flowed during 1974 as to how the job should be done, and it is perhaps not surprising that by the end of the year success should finally have been attained in the search for a method of labelling catecholamine receptors. Indeed, three groups have now reported binding assays which exhibit the characteristics predicted for $\beta$ adrenoceptors. Their success in the face of many previous disappointments illustrates the critical importance of choosing the right ligand and the right tissue for receptor binding studies. The three groups, Levitski et al. (Proc. natn. Acad. Sci. U.S.A., 71, 2773 and 4246; 1974) Lefkowitz et al. (Biochem. Biophys. res. Commun., 60, 703; 1974) and Aurbach et al. (Science, 186, 1223; 1974) all used high specific activity $\beta$-adrenoceptor antagonists as ligands, the compounds
Similarly they have significant infrared absorption intensity. In $\mathrm{HD}^{+}$the calculations suggest a dipole of $2.9 \times 10^{-30} \mathrm{C} \mathrm{m}(0.87 \mathrm{D})$ and a vibrational transition moment of $2.8 \times 10^{-31} \mathrm{C} \mathrm{m}(0.086 \mathrm{D})$.

Alongside this theoretical feature one must place the experimental success in the laboratory of Dixon and Woods (Phys. Rev. Lett., 34, 61 ; 1975) in the detection of a spectrum due to $\mathrm{CO}^{+}$. A pair of lines at 117692.55 and $118101.99 \mathrm{MHz}$ due to $J 1 / 2-1 / 2$ and $1 / 2-3 / 2$ respectively were observed. Special techniques with a sample length of $3.5 \mathrm{~m}$ included the discharge to form the ions and a signal averager to enhance the low signal-to-noise ratio through repetitive scanning. Because of the unpaired electron the spectrum is sensitive to magnetic fields and the detection of both lines enables the spin-rotation constant $\gamma_{0}$ to be evaluated as $272.96 \mathrm{MHz}$ and the rotational constant $B_{0}$ as 58983.13 $\mathrm{MHz}$. Although moderately accurate values were previously known from optical spectroscopy, these more precise values should be an excellent guide to radioastronomers who wish to seek for $\mathrm{CO}^{+}$.

being $\mathrm{DL}-{ }^{3} \mathrm{H}$-propanalol, $\mathrm{L}-{ }^{3} \mathrm{H}$-alprenolol and ${ }^{125} \mathrm{I}$-hydroxybenzylpindolol respectively. All of these drugs are very potent antagonists at $\beta$-adrenoceptors, with binding constants of the order of $10^{9} 1 \mathrm{~mol}^{-1}$. In addition all three groups used the membranes of avian or amphibian red blood cells as the source tissues for binding studies. Such cells are known to possess a catecholaminesensitive adenylate cyclase activity that is triggered by a $\beta$-adrenoceptor mechanism, although the physiological significance of such receptors in red blood cells remains unclear.

The binding of the labelled antagonists to the cell membranes was found to be saturable and a considerable proportion of the binding (as much as $80 \%$ in the case of Lefkowitz et al.) could be prevented by the addition of an excess of non-labelled propranalol or other known antagonists. Furthermore, the active 1--stereoisomers of the antagonist drugs propranalol and alprenolol were more than one hundred times as potent as the inactive $\mathrm{D}$-isomers in preventing binding of the labelled material. Binding could also be inhibited by other $\beta$-adrenoceptor antagonists, and by catecholamines such as isoprenaline, noradrenaline, adrenaline or dopamine, but not by $\alpha$ adrenoceptor antagonists such as phen- 